Pacific Journal of Mathematics

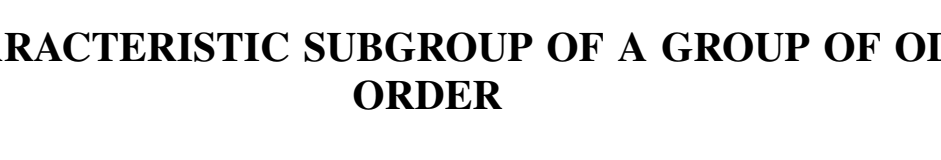




\section{A CHARACTERISTIC SUBGROUP OF A GROUP OF ODD ORDER}

\section{Z. Arad (Ardinast) and G. Glauberman}

Let $G$ be a finite solvable group of odd order. Suppose $p$ is a prime, $S$ is a Sylow $p$-subgroup of $G$, and $O_{p},(G)=1$. Let $J(S)$ be the Thompson subgroup of $S$. Then, by a result of the second author (Lemma 6), $Z(J(S)) \triangleleft G$.

The object of this paper is to generalize the above result by replacing the prime $p$ by a set of primes $\pi$.

We obtain the following results:

THEOREM 1. Let $G$ be a finite solvable group of odd order, $\pi$ be a set of primes, and $H$ be a Hall $\pi$-subgroup of $G$. Assume that $O_{\pi^{\prime}}(G)=1$. Then :

(a) for every $p \in \pi-\{3\}$ and $A \in \mathscr{A}(H), O_{p}(A) \subseteq O_{p}(G)$;

(b) the prime divisors of $d(H)$, of $|Z(J(H))|$, and of $|F(G)|$ coincide ;

(c) $d(G)=d(H)$; and

(d) $Z(J(G))=Z(J(H))$.

In particular, if $G \neq 1$, then $1 \subset Z(J(H)) \triangleleft G$.

Corollary. Suppose $G$ is a finite solvable group of odd order, $p$ is a prime, and $S$ is a Sylow p-subgroup of $G$. Assume that $O_{p^{\prime}}(G)=$ 1. Then $Z(J(S))=Z(J(G))$. Moreover, if $p \neq 3$, then $J(S)=J(G)=$ $J(F(G))$.

By the Odd Order Theorem of Feit and Thompson [1], Theorem 1 and its corollary apply to all finite groups of odd order. Since much of our argument requires only that $G$ be $\pi$-solvable and have an Abelian Sylow 2-subgroup, we obtain a related result:

Theorem 2. Suppose $\pi$ is a set of primes, $G$ is a finite $\pi$-solvable group, and $H$ is a Hall $\pi$-subgroup of $G$. Assume that $G$ has an Abelian Sylow 2-subgroup and that $O_{\pi^{\prime}}(G)=1$. Then:

(a) $O_{2}(G)=O_{2}\left(Z(J(G))=O_{2}(Z(J(H)))=O_{2}(H)\right.$;

(b) if $2 \notin \pi$, then for every $p \in \pi-\{3\}$ and $A \in \mathscr{A}(H), O_{p}(A) \subseteq$ $O_{p}(G)$

(c) if $2 \notin \pi$, then $Z(J(H)) \triangleleft G$; and

(d) if $2 \notin \pi$, then the prime divisors of $d(H)$, of $|Z(J(H))|$, and of $|F(G)|$ coincide. 
In particular, if $2 \notin \pi$ and $G \neq 1$, or if $O_{2}(G) \neq 1$, then there exists a nonidentity characteristic subgroup of $H$ that is a normal subgroup of $G$.

COROllary. Assume the hypothesis of Theorem 2 and assume that $2,3 \notin \pi$. Then $J(H)=J(F(G))$.

Some related results for groups with a nilpotent Hall $\pi$-subgroup were obtained by Schoenwaelder in [5].

All groups in this paper are assumed to be finite. Our notation is standard and taken mainly from [4]. In particular, let $G$ be a group. Then $F(G)$ denotes the Fitting subgroup of $G$ and $[A, B, C]$ denotes the triple commutator $[[A, B], C]$ of three subgroups $A, B, C$ of $G$. Moreover, $d(G)$ is the maximum of the orders of the Abelian subgroups of $G$. Let $\mathscr{A}(G)$ be the set of all Abelian subgroups of order $d(G)$ in $G$. (This is denoted by $A^{\prime}(G)$ in [4].) Then, as in [4], $J(G)$ is the subgroup of $G$ generated by $\mathscr{A}(G)$, that is, the Thompson subgroup of G.

For a prime power $q$, we will denote the finite field of $q$ elements by $G F(q)$. Let $p$ be a prime. Sometimes we will use $Z_{p}$ to denote $G F(p)$ considered as a field or as an additive group. We will often use without reference the elementary result that if $G$ is a group, $\pi$ a set of primes, and $H$ a normal subgroup of $G$, then $O_{\pi}(H) \subseteq O_{\pi}(G)$.

At times we shall assume one of the following hypotheses:

(a) $\pi$ is a set of primes

(b) $G$ is a $\pi$-solvable group

(c) $H$ is a Hall $\pi$-subgroup of $G$

$\left(\mathrm{H}_{2}\right) \quad$ (a) $\pi, G$, and $H$ satisfy $(H)$

(b) $G$ has an Abelian Sylow 2-subgroup.

(The concept of a $\pi$-solvable group is defined in $\$ 6.3$ of [4], in which it is proved that every $\pi$-solvable group possesses a Hall $\pi$-subgroup.)

\section{Preliminary results.}

Lemma 1. Suppose $p$ is a prime, $V$ is a finite nonidentity elementary Abelian additive p-group, and $A$ is an Abelian group of automorphisms of $V$. Regard $V$ as a vector space over $Z_{p}$. Assume that $A$ acts irreducibly on $V$ and that $A$ preserves some nondegenerate alternating bilinear form on $V$ into $Z_{p}$. Let $F$ be the ring of endomorphisms of $V$ generated by the elements of $A$.

Then:

(a) There exists a positive integer $k$ such that $|V|=p^{2 k}, F \cong$ $G F\left(p^{2 k}\right)$, and $|A|$ divides $1+p^{k}$. 
(b) Let $E$ be the unique subfield of $F$ that is isomorphic to $G F\left(p^{k}\right)$. Take $v_{0} \in V-\{0\}$ and let $W=v_{0} E$. Then for every nondegenerate alternating bilinear form $f$ on $V$ that is preserved by $A$,

$$
f\left(w, w^{\prime}\right)=0 \quad \text { for } \text { all } \quad w, w^{\prime} \in W .
$$

Proof. Let $F_{0}$ be the set (ring) of all endomorphisms of $V$ that commute with every element of $A$. We regard $Z_{p}$ as a subfield of $F_{0}$. As is well known, $F_{0}$ is a division algebra ([4], page 76) and, since it is finite, $F_{0}$ is a field. Clearly, $F$ is a subfield of $F_{0}$. Hence the multiplicative group $F-\{0\}$ is cyclic. As $A$ is a subgroup of $F-\{0\}, A$ is cyclic. Let $p^{m}=|V|$. We may regard $V$ as a vector space over $F$; then $V$ is a direct sum of 1-dimensional subspaces over $F$. As $A \subseteq F-\{0\}$ and $A$ acts irreducibly on $V, V$ is 1-dimensional over F. Therefore, $|\boldsymbol{F}|=|V|=p^{m}$.

Let $N$ be the set of all nondegenerate alternating bilinear forms on $V$ into $Z_{p}$ that are preserved by $A$. By hypothesis, $N$ is not empty. Hence $m$ is even. Choose a generator $\alpha$ of $A$. Define $g(x)$ to be the minimal polynomial of $\alpha$ over $Z_{p}$. Then $g(x)$ can be expressed as

$$
g(x)=\sum_{0 \leqq i \leqq m} a_{l} x^{i}
$$

where $a_{0}, \cdots, a_{m} \in Z_{p}$ and $a_{m}=1$. By the elementary theory of fields, the roots of $g(x)$ over $F$ are distinct and are precisely $\alpha, \alpha^{p}, \cdots, \alpha^{p^{m}}$ !.

Take some $f \in N$ and some $v \in V-\{0\}$. Let $v^{\prime}=v g\left(\alpha^{-1}\right)$. Then, for all $w \in V$,

$$
\begin{aligned}
f\left(v^{\prime}, w\right) & =\sum_{\iota} a_{i} f\left(v \alpha^{-\imath}, w\right)=\sum_{\iota} a_{i} f\left(v, w \alpha^{\iota}\right) \\
& =f(v, w g(\alpha))=0 .
\end{aligned}
$$

Since $f$ is not degenerate, $v^{\prime}=0$. As $v$ was chosen arbitrarily, $g\left(\alpha^{-1}\right)=$ 0 . Hence, $\alpha^{-1}=\alpha^{p^{\prime}}$ for some $i$ such that $0 \leqq i \leqq m-1$. If $i=0$, then $\alpha^{2}=1$, contrary to the fact that $m \geqq 2$ and $\alpha \neq \alpha^{p}$. Therefore, $1 \leqq i \leqq$ in - 1. Now

$$
\alpha=\left(\alpha^{-1}\right)^{-1}=\left(\alpha^{p^{\prime}}\right)^{-1}=\left(\alpha^{-1}\right)^{p^{\prime}}=\alpha^{p^{2 i}} .
$$

Since $\alpha$ generates $F$ and $F \cong G F\left(p^{m}\right), 2 i$ is a multiple of $m$. Consequently, $i=\frac{1}{2} m$. Let $k=\frac{1}{2} m$. Then $\alpha^{-1}=\alpha^{p^{k}}$, and $\alpha^{1+p^{k}}=$ 1. This proves (a). 
Let $\delta=\alpha+\alpha^{-1}$. Since

$$
\delta^{p^{k}}=\alpha^{p^{k}}+\alpha^{p^{2 k}}=\alpha+\alpha^{p^{k}}=\delta,
$$

$\delta \in E$. Since $\alpha$ generates $F$ over $Z_{p}$, it follows that $\alpha, \alpha^{p}, \cdots, \alpha^{p^{2 k-1}}$ form a basis of $F$ over $Z_{p}$. Hence $\delta, \delta^{p}, \cdots, \delta^{p^{k-1}}$ are distinct. So, $\delta$ generates $E$ over $Z_{p}$ and $\delta, \delta^{p}, \cdots, \delta^{p^{k-1}}$ form a basis of $E$ over $Z_{p}$, that is,

$$
\alpha+\alpha^{-1}, \alpha^{p}+\alpha^{-p}, \cdots, \alpha^{p^{k-1}}+\alpha^{-p^{k-1}}
$$

is a basis of $E$ over $Z_{p}$.

Take $f \in N$ and $w, w^{\prime} \in W$ as in (b). If $w=0$, then $f\left(w, w^{\prime}\right)=$ 0 , as desired. Assume that $w \neq 0$. Then there exists $\beta \in E$ such that $w^{\prime}=w \beta$. Take $b_{0}, b_{1}, \cdots, b_{k-1} \in E$ such that

$$
\sum_{0 \leq i \leq k-1} b_{i}\left(\alpha^{p^{i}}+\alpha^{-p^{i}}\right)=\beta
$$

For $i=0, \cdots, k-1$,

$$
\begin{aligned}
f\left(w, w\left(\alpha^{p^{\prime}}+\alpha^{-p^{\prime}}\right)\right) & =f\left(w, w \alpha^{p^{i}}\right)+f\left(w, w \alpha^{-p^{i}}\right) \\
& =f\left(w, w \alpha^{p^{\prime}}\right)+f\left(w \alpha^{p^{\prime}}, w\right)=0,
\end{aligned}
$$

since $f$ is an alternating form. Hence,

$$
f\left(w, w^{\prime}\right)=f(w, w \beta)=\sum_{0 \leqq i \leqq k-1} b_{\imath} f\left(w, w\left(\alpha^{p^{\prime}}+\alpha^{-p^{\prime}}\right)\right)=0
$$

as desired. This completes the proof of (b) and thus of Lemma 1.

Lemma 2. Suppose $p$ is a prime, $B$ is a finite, non-Abelian p-group, and $A$ is an Abelian group of automorphisms of $B$. Assume that $A$ acts irreducibly on $B / \Phi(B)$ and that $O_{p^{\prime}}(A)$ acts trivially on $\Phi(B)$.

Then:

(a) there exists a positive integer $k$ such that $|B / \Phi(B)|=p^{2 k}$;

(b) $|A|$ divides $1+p^{k}$; and

(c) $B$ contains an Abelian subgroup $B_{0}$ such that $B_{0} \supseteq \Phi(B)$ and $\left|B_{0} / \Phi(B)\right|=p^{k}$.

Proof. For convenience in notation, we embed $A$ and $B$ in the natural manner in their semi-direct product $A B$.

Let $A_{p}=O_{p}(A), A^{*}=O_{p^{\prime}}(A)$, and $V=B / \Phi(B)$. Since $A$ acts 
irreducibly on $V, A / C_{A}(V)$ acts faithfully and irreducibly on $V$. We may regard $V$ as a vector space over $Z_{p}$. By [4], Theorem 3.1.3, page 62 ,

$$
A_{p} C_{A}(V) / C_{A}(V)=O_{p}\left(A / C_{A}(V)\right)=1
$$

Hence

$$
A_{p} \subseteq C_{A}(V) \text { and } A^{*} \text { acts irreducibly on } V \text {. }
$$

Since $B$ is not Abelian, $B$ is not cyclic. Therefore, $|V|=$ $|B / \Phi(B)| \geqq p^{2}$. It follows that $1 \neq\left[V, A^{*}\right]$ and therefore that

$$
\left[V, A^{*}\right]=V .
$$

Consequently, $B=\left[B, A^{*}\right] \Phi(B)$. By [4], page 173 .

$$
B=\left[B, A^{*}\right] \text {. }
$$

By (1) and the hypothesis of this lemma,

$$
\left[A_{p}, B, A^{*}\right] \subseteq\left[\Phi(B), A^{*}\right]=1 \quad \text { and }\left[A^{*}, A_{p}, B\right]=[1, B]=1 \text {. }
$$

Therefore, by (3) and the Three Subgroups Lemma ([4], page 19),

$$
1=\left[B, A^{*}, A_{p}\right]=\left[B, A_{p}\right] \text {. }
$$

As $A_{p} \subseteq$ Aut $B, A_{p}=1$. Hence $A$ is a $p^{\prime}$-group and $A=A^{*}$. By a theorem of Burnside ([4], page 174),

$$
\text { A acts faithfully on } V \text {. }
$$

Since $C_{A B}(\Phi(B))$ is a normal subgroup of $A B$ that contains $A$, (3) yields that $C_{A B}(\Phi(B))$ contains $B$. Therefore, $\Phi(B) \subseteq Z(B)$. Since $B$ is not Abelian and $B^{\prime} \subseteq \Phi(B) \subseteq Z(B), B$ has nilpotence class two. By an easy calculation, $[x, y]^{p}=\left[x^{p}, y\right]=1$ for all $x, y \in B$. Thus

$$
B^{\prime} \text { is an elementary Abelian group. }
$$

Take any subgroup $C$ of index $p$ in $B^{\prime}$. Let $\phi$ be an isomorphism of $B^{\prime} / C$ onto the additive group of $Z_{p}$. Since $\Phi(B) \subseteq Z(B)$, the mapping $f: V \times V \rightarrow Z_{p}$ given by

$$
f(x \Phi(B), y \Phi(B))=\phi([x, y] C)
$$


is a well-defined, nonzero, alternating bilinear form on $V$ into $Z_{p}$. As $A$ acts trivially on $B^{\prime}, A$.preserves $f$. Therefore, $A$ preserves the radical of $f$, that is, the group $R / \Phi(B)$, where

$$
R \supseteq \Phi(B) \supset C \text { and } R / C=Z(B / C) .
$$

As $R / \Phi(B) \subset V$ and $A$ acts irreducibly on $V, \quad R / \Phi(B)=$ 1. Consequently, $f$ is a nondegenerate form. By (4) and Lemma 1, there exists a positive integer $k$ such that $|V|=p^{2 k}$ and $|A|$ divides $1+p^{k}$. This yields (a) and (b).

Take $E$ and $W$ as in Lemma 1(b). Define a subgroup $B_{0}$ of $B$ such that $B_{0} \supseteq \Phi(B)$ and $B_{0} / \Phi(B)=W$. Then

$$
\left|B_{0} / \Phi(B)\right|=|W|=|E|=p^{k} .
$$

Suppose $B_{0}^{\prime} \neq 1$. Then, by (5), there exists a subgroup $C^{*}$ of index $p$ in $B^{\prime}$ such that $B_{0}^{\prime} \notin C^{*}$. For convenience in notation, we will assume that $C^{*}$ is the group $C$ chosen above. Take a form $f$ as above. Take $x, y \in B_{0}$ such that $[x, y] \notin C$. Then

$$
f(x \Phi(B), y \Phi(B))=\phi([x, y] C) \neq 0,
$$

contrary to Lemma $1(\mathrm{~b})$. This contradiction proves that $B_{0}^{\prime}=1$ and hence completes the proof of (c) and of Lemma 2.

Lemma 3. Assume $(\mathrm{H})$ and assume that $O_{\pi^{\prime}}(G)=1$. Then :

(a) $C_{G}(F(G)) \subseteq F(G)$, and

(b) if $A$ is a subgroup of $A$ ut $G$ that fixes every element of $F(G)$ and if $|A|$ and $|G|$ are relatively prime, then $A=1$.

Proof. (a) Let $N=O_{\pi}(G)$ and $C=C_{G}(F(G))$. Then $N$ is a solvable group. Clearly, $F(N)=F(G)$. By [4], Theorem 6.3.2, $C_{G}(N) \subseteq N$.

Suppose $x$ is a $\pi^{\prime}$-element in $C$. Let $L=\langle N, x\rangle$. Then

$$
N=O_{\pi}(L) \quad \text { and } \quad\left[N, O_{\pi^{\prime}}(L)\right] \subseteq N \cap O_{\pi^{\prime}}(L)=1 .
$$

Since $C_{G}(N) \subseteq N$, it follows that $O_{\pi^{\prime}}(L)=1$. Hence $F(N)=$ $F(L)$. Since $L$ is solvable,

$$
x \in C \cap L=C_{L}(F(L)) \subseteq F(L)=F(N),
$$

by [4], page 218 . Therefore, $x=1$. 
Thus, $C$ is a $\pi$-group. Since $C \triangleleft G, C \subseteq O_{\pi}(G)=N$. By [4], page 218 again, $C=C_{N}(F(N)) \subseteq F(N)$.

(b) Embed $A$ and $G$ in their semi-direct product $A G$. Let $B=O_{\pi^{\prime}}(A G)$. Since $B \cap G \subseteq O_{\pi^{\prime}}(G)=1,|B|$ divides $|A G / G|$, that is, $|B|$ divides $|A|$. Since $|A|$ and $|G|$ are relatively prime and

$$
|A /(A \cap B)|
$$

divides $|A G / B|, B \subseteq A$. However,

$$
[G, B] \subseteq\left[G, O_{\pi^{\prime}}(A G)\right] \subseteq O_{\pi^{\prime}}(G)=1 .
$$

As $B$ is a group of automorphisms of $G, B=1$. Hence $F(A G)=$ $F(G)$. By (a), $A \subseteq F(G)$. Therefore, $A=1$.

Lemma 4. Assume (H). Suppose $p \in \pi, O_{\pi^{\prime}}(G)=1$, and $T$ is a p-subgroup of $O_{p^{\prime} p}(G)$ that centralizes $F\left(O_{p^{\prime}}(G)\right)$. Then $T \subseteq O_{p}(G)$.

Proof. Let $K=O_{p},(G)$. Apply Lemma 3 with $K$ in place of $G$ and $T / C_{T}(K)$ in place of $A$. We obtain the conclusion that $T / C_{T}(K)=$ 1 , in other words, $T$ centralizes $K$. Let $R$ be a Sylow $p$-subgroup of $O_{p^{\prime}, p}(G)$ that contains $T$. Let $T^{*}=C_{R}(K)$. Then $O_{p^{\prime}, p}(G)=K R$ and $T^{*}$ is normalized by $K$ and by $R$. Hence $T^{*} \triangleleft K R$ and

$$
T \subseteq T^{*} \subseteq O_{p}(K R) \subseteq O_{p}(G)
$$

We also use the following result of J. Thompson, whose proof is sketched in the remark on page 164 of [3]:

Theorem of ThOMPSon. Suppose $p$ is an odd prime, $G$ is a p-solvable group, and $S$ is a Sylow p-subgroup of $G$. Assume that $O_{p^{\prime}}(G)=1$. Assume also that $G$ satisfies one of the following conditions :

(i) $p \geqq 7$;

(ii) $p=5$ and $G$ has an Abelian Sylow 2-subgroup.

Then $J(S) \subseteq O_{p}(G)$.

Lemma 5. Assume $\left(\mathrm{H}_{2}\right)$. Suppose $p \in \pi, \quad S$ is a Sylow p-subgroup of $G$, and $A \in \mathscr{A}(S)$. Assume that $p \geqq 5$ and that $A$ centralizes $F\left(O_{p},(G)\right)$. Then $A \subseteq O_{p}(G)$.

Proof. Let $K=O_{p^{\prime}}(G)$. Note that $G$ is $p$-solvable. By the Theorem of Thompson, 


$$
A K / K \subseteq O_{p}(G / K)=O_{p^{\prime}, p}(G) / K
$$

Hence $A \subseteq O_{p^{\prime}, p}(G)$. By Lemma 4, $A \subseteq O_{p}(G)$, as desired.

LemMa 6. Suppose $p$ is an odd prime, $G$ is a p-solvable group, and $S$ is a Sylow p-subgroup of $G$. If $p=3$, assume also that $G$ has an Abelian Sylow 2-subgroup. Then

$$
O_{p},(G) Z(J(S)) \triangleleft G .
$$

Proof. Let $K=O_{p^{\prime}}(G), G^{*}=G / K$, and $S^{*}=S K / K$. Then $O_{p^{\prime}}\left(G^{*}\right)=1$ and $S^{*}$ is a Sylow $p$-subgroup of $G^{*}$. From the hypothesis, $G^{*}$ must be $p$-constrained and $p$-stable. By a theorem of the second author ([4], pages 268-269 and 279, or [2], Theorem A), $Z\left(J\left(S^{*}\right)\right) \triangleleft G^{*}$. Since

$$
Z\left(J\left(S^{*}\right)\right)=Z(J(S)) K / K
$$

the result follows.

The next result can be easily verified by calculation. It is a special case of Lemma 10.1, page 1131, of [2].

LemMA 7. Let $K$ be a group of linear transformations on a finite-dimensional vector space $V$ over a field $F$. Let $V^{*}$ be the dual space of $V$ over $F$ and let $K$ act on $V^{*}$ in the natural manner, i.e.,

$$
f^{g}(v)=f\left(v^{g^{-1}}\right), \quad \text { for } \quad f \in V^{*}, g \in K, v \in V
$$

Let $T$ be the set of all ordered triples $(v, f, \alpha)$ for $v \in V, f \in V^{*}$, $\alpha \in F$. Define multiplication on $T$ by the rule

$$
\left(v_{1}, f_{1}, \alpha_{1}\right)\left(v_{2}, f_{2}, \alpha_{2}\right)=\left(v_{1}+v_{2}, f_{1}+f_{2}, \alpha_{1}+\alpha_{2}-f_{1}\left(v_{2}\right)\right) \text {. }
$$

For each $g \in K$, define a mapping $M(g)$ of $T$ into itself by

$$
(v, f, \alpha)^{M(g)}=\left(v^{g}, f^{g}, \alpha\right) .
$$

Then:

(a) $T$ forms a group under multiplication;

(b) for $(v, f, \alpha),\left(v_{1}, f_{1}, \alpha_{1}\right)$ and $\left(v_{2}, f_{2}, \alpha_{2}\right)$ in $T$,

$$
(v, f, \alpha)^{-1}=(-v,-f,-f(v)-\alpha)
$$




$$
\left[\left(v_{1}, f_{1}, \alpha_{1}\right),\left(v_{2}, f_{2}, \alpha_{2}\right)\right]=\left(0,0, f_{2}\left(v_{1}\right)-f_{1}\left(v_{2}\right)\right) ; \quad \text { and }
$$

(c) $M$ is an isomorphism of $K$ into the automorphism group of $T$.

\section{Some Properties of $\mathscr{A}(G)$.}

Proposition 1. Suppose $G$ is group, $A \in \mathscr{A}(G), B$ is a nilpotent subgroup of $G$, and $A$ normalizes $B$. Assume that $B$ has an Abelian Sylow 2-subgroup and that either $|A|$ is odd or $B$ is Abelian. Then $A B$ is nilpotent.

Proof. Assume that the result is false, that $G$ is a counter-example of minimal order, and that, within $G, B$ has minimal order.

Clearly, $G=A B$ and $G \supset F(G) \supseteq B$. Therefore, $A \not \subset F(G)$. For some prime $p, \quad O_{p}(A) \notin \subset F(G)$. Let $A_{p}=O_{p}(A)$. Then $A_{p} \notin O_{p}(G)$. Hence $A_{p} B_{p} \not G G$. Since $A$ normalizes $A_{p} B_{p}, B$ does not. Consequently, there exists a prime $q$ such that $O_{q}(B)$ does not normalize $A_{p} B_{p}$. Let $B_{q}=O_{q}(B)$. Then $B_{q}$ does not centralize $A_{p} B_{p}$ and therefore does not centralize $A_{p}$. Thus $A B_{q}$ is not nilpotent. By the minimal choice of $B, B=B_{q}$.

Let $A^{*}=O_{q^{\prime}}(A)$ and $V=B / B^{\prime}$. Then $A^{*}$ does not centralize $B$. By [4], page 174, $A^{*}$ does not centralize $V$. By the minimal choice of $B$,

$$
A^{*} \text { centralizes } \Phi(B) \text {. }
$$

From [4], page 177, $V=C_{V}\left(A^{*}\right) \times\left[V, A^{*}\right] . \quad$ By the minimal choice of $B$,

$$
V=\left[V, A^{*}\right] \text { and } C_{V}\left(A^{*}\right)=1 \text {. }
$$

Let $W$ be a minimal $A$-invariant subgroup of $V$. Then $W$ is elementary Abelian. Since $C_{W}\left(A^{*}\right) \subseteq C_{V}\left(A^{*}\right)=1$, the minimal choice of $V$ yields that $V=W$. Hence $\Phi(B) \subseteq B^{\prime} \subseteq \Phi(B)$. Consequently,

(8) $\quad B^{\prime}=\Phi(B)$ and $A$ acts irreducibly and nontrivially on $B / B^{\prime}$.

Let $C=C_{A}(B)$ and $n=|A / C|$. Then $A / C$ acts faithfully as a group of automorphisms of $B$. By (8),

$$
C \cap B \subseteq B^{\prime}
$$

Take $B_{1} \in \mathscr{A}(B)$. Since $C B_{1}$ is Abelian and $A \in \mathscr{A}(G)$, 


$$
|A| \geqq\left|C B_{1}\right|=|C|\left|B_{1}\right| / C \cap B_{1}|\geqq| C|| B_{1}|/| B^{\prime} \mid,
$$

by (9). Hence

$$
n=|A / C| \geqq\left|B_{1}\right| /\left|B^{\prime}\right|=d(B) /\left|B^{\prime}\right| \text {. }
$$

Suppose first that $B$ is Abelian. Then $B^{\prime}=1$ and $d(B)=$ $|B|$. For every $a \in A-C, C_{B}(a) \subset B$ and $C_{B}(a) \triangleleft A B$; by (8), $C_{B}(a)=1$. Hence every non-identity element of $A / C$ acts in a fixedpoint-free manner on $B$, and

$$
|A / C| \leqq|B-\{1\}|<|B|=d(B) /\left|B^{\prime}\right| \text {. }
$$

However, this contradicts (10).

Thus $B$ is not Abelian. By hypothesis,

$$
q \text { is an odd prime and }|A| \text { is odd. }
$$

By (7) and (8), $A$ and $B$ satisfy the hypothesis of Lemma 2. Take $k$ and $B_{0}$ as in Lemma 2 . Then

$$
\left|B / B^{\prime}\right|=q^{2 k}, n \text { divides } 1+q^{k}, B_{0} \text { is abelian, and }\left|B_{0} / B^{\prime}\right|=q^{k} .
$$

Therefore, by $(10), n \geqq d(B) /\left|B^{\prime}\right| \geqq\left|B_{0} / B^{\prime}\right|=q^{k}$. Since $n$ divides $1+q^{k}, n=1+q^{k}$. But this is impossible, by (11). This contradiction completes the proof of Proposition 1.

Proposition 2. Assume $\left(H_{2}\right)$. Suppose $O_{\pi^{\prime}}(G)=1$. Then

$$
O_{2}(G)=O_{2}(H)=O_{2}(Z(J(H)))=O_{2}(Z(J(G))) .
$$

Proof. Let $K=O_{2}(Z(J(H)))$ and $N=O_{\pi}(G)$. Then $N$ is a solvable group. By $\left(\mathrm{H}_{2}\right), K$ centralizes $\mathrm{O}_{2}(G)$. For every odd prime $p$,

$$
O_{p}(G) \subseteq O_{p}(H) \subseteq C_{G}\left(O_{2}(H)\right) \subseteq C_{G}(K)
$$

Hence $K$ centralizes $F(G)$. By Lemma $3, \quad K \subseteq C_{G}(F(G)) \subseteq$ $F(G)$. So $K \subseteq O_{2}(F(G))=O_{2}(G)$.

On the other hand, let $A \in \mathscr{A}(H)$ and $B=O_{2}(G)$. By Proposition $1, A B$ is nilpotent. Therefore, $O_{2}(A)$ centralizes $B$. By $\left(\mathrm{H}_{2}\right), A$ centralizes $B$. Hence $B \subseteq C_{H}(A)=A$. Thus $B \subseteq Z(J(H))$ and $B \subseteq$ $K$. Consequently, $B=K$, as desired. Since $\pi, H$, and $H$ satisfy $\left(\mathrm{H}_{2}\right)$, we obtain as a special case that $K=O_{2}(H)$. 
A similar argument with $A \in \mathscr{A}(G)$ and $B=O_{2}(G)=K$ shows that $K \subseteq Z(J(G))$. Hence

$$
K \subseteq O_{2}(Z(J(G))) \subseteq O_{2}(G)=K
$$

So $K=O_{2}(Z(J(G)))$.

Proposition 3. Assume $\left(\mathrm{H}_{2}\right)$. Suppose $p \in \pi$ and $A \in \mathscr{A}(H)$. Assume that $O_{\pi^{\prime}}(G)=1, d(H)$ is odd, and $p \geqq 5$. Then $O_{p}(A) \subseteq$ $O_{p}(G)$.

Proof. We use induction on the order of $G$. Let $A_{p}=O_{p}(A)$, $T=O_{p}(G), K=O_{p, p}(G)$ and $G^{*}=A K$, and $H^{*}=A(H \cap K)$. Then $H \cap K$ is a Hall $\pi$-subgroup of $K$ and $H^{*}$ is a Hall $\pi$-subgroup of $G^{*}$.

Suppose $G^{*} \subset G$. Since $A \subseteq H^{*}, d\left(H^{*}\right)=d(H)$. By induction, $A_{p} \subseteq O_{p}\left(G^{*}\right)$. Hence

$$
\left[K, A_{p}\right] \subseteq K \cap O_{p}\left(G^{*}\right) \subseteq O_{p}(K)=T
$$

Therefore, $A_{p} T / T \subseteq C_{G / T}(K / T)$. By [4], page 228, $C_{G / T}(K / T) \subseteq$ $K / T$. Consequently, $A_{p} \subseteq K$. So,

$$
A_{p} \subseteq K \cap O_{p}\left(G^{*}\right)=O_{p}(K)=T,
$$

as desired.

Suppose $G^{*}=G$. Then $A_{p} T$ is a Sylow $p$-subgroup of $G$. Let $A^{*}=O_{p^{\prime}}(A)$. By hypothesis, $|A|$ is odd. By Proposition $1, A T$ is nilpotent. Therefore, $A^{*}$ centralizes $T$ and hence $A_{p} T$. For every Abelian subgroup $B$ of $A_{p} T, A^{*} B$ is Abelian and

$$
\left|A^{*}\right|\left|A_{p}\right|=|A| \geqq|A * B|=|A *||B| \text {. }
$$

Hence $A_{p} \in \mathscr{A}\left(A_{p} T\right)$. By Proposition 1, $A F\left(O_{p^{\prime}}(G)\right)$ is nilpotent. Then $A_{p}$ centralizes $F\left(O_{p}(G)\right)$. 'By Lemma 5, $A_{p} \subseteq O_{p}(G)$, as desired.

Proposition 4. Assume $\left(\mathrm{H}_{2}\right)$. Suppose $\pi$ is a set of odd primes and $O_{\pi^{\prime}}(G)=1$.

Let $K=C_{G}\left(O_{3}(G)\right)$. For every $p \in \pi$ and $A \in \mathscr{A}(H)$, let $A_{p}=$ $O_{p}(A)$. Define $d_{3}$ to be the maximum of $|C|$ for all Abelian 3subgroups $C$ of $H \cap K$ and define $\mathscr{A}_{3}$ to be the set of all Abelian 3-subgroups of order $d_{3}$ in $H \cap K$. Let $S$ be any Sylow 3-subgroup of K. Then: 
(a) $\left\{A_{p} \mid A \in \mathscr{A}(H)\right\}=\mathscr{A}\left(O_{p}(G)\right)$, for every prime $p \geqq 5$;

(b) $\left\{A_{3} \mid A \in \mathscr{A}(H)\right\}=\mathscr{A}_{3}$;

(c) $O_{p}(Z(J(H)))=Z\left(J\left(O_{p}(G)\right)\right)$, for every prime $p \geqq 5$; and

(d) $O_{3}(Z(J(H)))=Z(J(S)) \triangleleft G$ and $d_{3}=d(S)$.

Proof. Note that $d(H)$ is odd.

(a) Assume $p \geqq 5$. Let $A \in \mathscr{A}(H)$. Let $A^{*}=O_{p^{\prime}}(A)$ and $M=$ $O_{p}(G)$. By Proposition 3, $A_{p} \subseteq M$. By Proposition $1, A^{*}$ centralizes $M$. Hence, for every Abelian subgroup $B$ of $M, A^{*} \times B$ is Abelian. Therefore, $\left|A_{p}\right|=d(M)$, and $A^{*} \times B \in \mathscr{A}(H)$ for every $B \in \mathscr{A}(M)$. This proves (a).

(b) Suppose $A \in \mathscr{A}(H)$. By Proposition $1, A F(G)$ is nilpotent. Hence, $A_{3}$ centralizes $F\left(O_{3}(G)\right)$. Since

$$
O_{\pi^{\prime}}\left(O_{3^{\prime}}(G)\right) \subseteq O_{\pi^{\prime}}(G)=1,
$$

$A_{3}$ centralizes $O_{3^{\prime}}(G)$, by Lemma 3. By (a), $O_{3^{\prime}}(A) \subseteq O_{3^{\prime}}(G)$. Now (b) follows by an argument similar to that of (a).

(c) This follows immediately from (a).

(d) Assume first that $K$ is a $3^{\prime}$-group. Then $\mathscr{A}_{3}=\{1\}$ and $S=$ 1. Since $Z(J(H)) \subseteq A$ for every $A \in \mathscr{A}(H), \quad O_{3}(Z(J(H)))=1=$ $Z(J(S))$, as desired.

Now assume that $K$ is not a $3^{\prime}$-group. Then $S \neq 1$. Let $T=$ $O_{3}(Z(J(H)))$ and $U=Z(J(S))$. By Lemma 6, $U O_{3^{\prime}}(K) \triangleleft K$. Since $O_{3^{\prime}}(K) \subseteq O_{3}(G)$ and $K=C_{G}\left(O_{3}(G)\right)$,

$$
U O_{3^{\prime}}(K)=U \times O_{3^{\prime}}(K) .
$$

Hence

$$
1 \subset U=O_{3}\left(U O_{3^{\prime}}(K)\right)<K \text {. }
$$

As $O_{\pi^{\prime}}(G)=1$ and $1 \subset U \subseteq O_{3}(K) \subseteq O_{3}(G), 3 \in \pi$.

Suppose $A \in \mathscr{A}(H)$. By (b), $A_{3} \subseteq H \cap K$. Let $A^{*}=O_{3}(A)$ and let $S^{*}$ be a Sylow 3-subgroup of $H \cap K$ that contains $A_{3}$. Since $K \varangle G$ and $3 \in \pi, H \cap K$ is a Hall $\pi$-subgroup of $K$ and $S^{*}$ is a Sylow 3 -subgroup of $K$. As $S^{*}$ and $S$ are conjugate in $K$, (12) yields that

$$
U=Z J\left(S^{*}\right) \text {. }
$$

By (a), $A^{*} \subseteq O_{3^{\prime}}(G)$. Therefore, $S^{*}$ centralizes $A^{*}$. Since $A=$ $A_{3} \times A^{*}, \quad A_{3} \in \mathscr{A}\left(S^{*}\right)$ and $d_{3}=\left|A_{3}\right|=d\left(S^{*}\right)=d(S)$. By (13), $U \subseteq$ $A_{3} \subseteq A$. As $A$ is an arbitrary element of $\mathscr{A}(H), U \subseteq Z(J(H))$. So, 
$U \subseteq T$. On the other hand, $T \subseteq A_{3}$ for every $A \in \mathscr{A}(H)$. Consequently, $T \subseteq B$ for every $B \in \mathscr{A}(S)$, by (b), and hence $T \subseteq U$. Thus $T=U$.

By (12), $U=Z(J(R))$ for every Sylow 3-subgroup $R$ of $K$. Therefore, $U$ is a characteristic subgroup of $K$ and hence a normal subgroup of $G$. This completes the proof of (d) and thus of Proposition 4.

\section{Proof of Theorems.}

We first prove Theorem 2. Parts (a) and (b) follow directly from Proposition 2 and 3 . Since

$$
Z(J(H))=\left\langle O_{p}(Z(J(H))) \mid p \in \pi\right\rangle
$$

(c) follows from Proposition 4. To prove (d), assume $2 \notin \pi$ and let $\pi_{1}, \pi_{2}$, and $\pi_{3}$ be the sets of prime divisors of $|Z(J(H))|, d(H)$, and $|F(G)|$ respectively. Since $Z(J(H)) \subseteq A$ for every $A \in \mathscr{A}(H)$,

$$
\pi_{1} \subseteq \pi_{2}
$$

Take $S$ as in Proposition 4. Note that $O_{3}(G) \subseteq K$, so $O_{3}(G) \subseteq$ $S$. Therefore,

$$
3 \in \pi_{1} \text { if and only if } 3 \in \pi_{3},
$$

by Proposition 4(d). By parts (b) and (d) of Proposition 4,

$$
\text { if } 3 \in \pi_{2} \text {, then } \mathscr{A}_{3} \neq\{1\}, S \neq 1 \text {, and } 3 \in \pi_{3} \text {. }
$$

Now (14), (15), and (16) yield that 3 belongs to all of $\pi_{1}, \pi_{2}$, and $\pi_{3}$ or none of them. Parts (a) and (c) of Proposition 4 yield an analogous statement for each prime greater than 3 . This completes the proof of Theorem 2.

Finally, we prove Theorem 1. For each prime $p$, define $d(p)$ to be the highest power of $p$ that divides $d(H)$. Let $\sigma$ be the set of all odd primes. We may and will assume that $2 \notin \pi$. Define $d_{3}$ as in Proposition 4.

Parts (a) and (b) of Theorem 1 are special cases of Theorem 2. By Proposition 4,

$$
d(3)=d_{3} \text { and } d(p)=d\left(O_{p}(G)\right) \text { for every prime } p>3 .
$$

Hence $d(H)=d_{3} \Pi_{p>3} d\left(O_{p}(G)\right)$. Thus, $d(H)$ does not depend on the 
choice of $\pi$, provided that $\pi \subseteq \sigma$ and $O_{\pi^{\prime}}(G)=1$. As $G$ is a Hall $\sigma$-subgroup of $G, d(G)=d(H)$. A similar argument from Proposition 4 shows that $Z(J(G))=Z(J(H))$.

\section{Some examples.}

EXAmPle 1. Let $q$ be a power of a prime $p$. Let $E=G F(q)$ and $F=G F\left(q^{2}\right)$. Take a fixed element $\mu$ of $F-E$ and define $B$ to be the set of all ordered pairs of the form $(\alpha, \beta)$ for $\alpha \in F$ and $\beta \in E$. Define multiplication on $B$ by the rule

$$
(\alpha, \beta)(\gamma, \delta)=\left(\alpha+\gamma, \beta+\delta+\alpha \mu \gamma^{q}+\alpha^{q} \mu^{q} \gamma\right)
$$

By calculation one may show that $B$ is a group of order $q^{3}$. Moreover, for $(\alpha, \beta) \in B$,

$$
C_{B}((\alpha, \beta))=\{(\gamma, \delta) \mid \gamma \in \alpha E, \delta \in E\} \quad \text { if } \quad \alpha \neq 0
$$

By further calculations,

$$
d(B)=q^{2} \text { and } B^{\prime}=\Phi(B)=Z(B)=\{(0, \beta) \mid \beta \in E\} .
$$

Take a nonzero element $\gamma$ of $F$ that has multiplicative order $q+1$. The mapping $\phi: B \rightarrow B$ given by

$$
\phi((\alpha, \beta))=(\alpha \gamma, \beta)
$$

is an automorphism of $B$ that has order $q+1$. Let $G$ be the semidirect product of $B$ by $\langle\phi\rangle$. Embed $\langle\phi\rangle$ and $B$ in $G$ in the natural manner. Let $A=\left\langle\phi, B^{\prime}\right\rangle$. Then $A$ is Abelian and $\left.|A|=(q+1) q\right\rangle$ $d(B)$, by (17). A short argument shows that $C_{G}(b) \subseteq B$ for every $b \in B-B^{\prime}$ and that $d(G)=(q+1) q$ and $A \in \mathscr{A}(G)$.

The group of automorphisms $\langle\phi\rangle$ yields an example of the "extreme' cases of Lemmas 1 and 2 , that is, $|\langle\phi\rangle|=1+p^{k}$ for $p^{k}=$ $q$. Since $B$ is nilpotent and $A B$ is not nilpotent, $G$ violates the conclusion of Proposition 1 ; here, $B$ is not Abelian, $B$ is a 2-group if $p=2$, and $|A|$ is even if $p \neq 2$.

Let $\pi$ be the set of all prime divisors of $|G|$ and let $H=G$. Then $G$ violates various conclusions of Theorems 1 and 2 . For every $r \in \pi-\{p\}, O_{r}(A) \neq 1$ and $O_{r}(G)=1$, although it is possible that $r \geqq$ 5. Furthermore, every element of $\pi$ divides $d(G)$, but $p$ is the only prime divisor of $|Z(J(G))|$ and is the only prime divisor of $|F(G)|$. Note, however, that obviously $Z(J(H)) \triangleleft G$. 
EXAMPLE 2. Let $F=G F(3)$ and let $V$ be a 3-dimensional vector space over $F$. Then there exists a group $K$ of linear transformations of $V$ over $F$ such that $K$ has order 39 and is not cyclic. Define $T$ and $M$ as in Lemma 7, and define $K$ to be an operator group on $T$ by the rule $t^{g}=t^{M(g)}$ for $t \in T, g \in K$.

Let $G$ be the semi-direct product of $T$ by $K$ and embed $T$ and $K$ in $G$ in the natural manner. Let $\pi$ be $\{3\}$ and $H$ be a Sylow 3-subgroup of $G$. Then $T$ is an extra-special group of order $3^{7}, T=F(G)$, and $d(H)=d(T)=3^{4}$. There exists $A \in \mathscr{A}(H)$ such that $A \not \subset T$. Then $A=O_{3}(A) \notin \subset O_{3}(G)=T$. Thus, part (a) of Theorem 1, part (b) of Theorem 2, and the corollary of Theorem 2 cannot be extended to include the case in which $p=3$.

Example 3. Here $G$ is defined as in Example 2 except that $K$ is taken to be isomorphic to the alternating group of degree 4 .

\section{REFERENCES}

1. W. Feit and J. G. Thompson, Solvability of groups of odd order, Pacific J. Math., 13 (1963), $755-1029$.

2. G. Glauberman, A characteristic subgroup of a p-stable group, Canad. J. Math., 20 (1968), $1101-1135$.

3. - Prime-power factor groups of finite groups, Math. Zeit., 107 (1968), 159-172.

4. _ Finite Groups, Harper and Row, New York (1968).

5. U. Schoenwaelder, Normale Komplemente zu nilpotenten Hall-Untergruppen, Archiv der Math., 19 (1968), 361-377.

Received December 26, 1973. The first author wishes to express his gratitude to his dissertation advisor, Professor M. Herzog, of the Department of Mathematical Sciences, Tel-Aviv University, for his guidance and concern. During the preparation of this paper, the second author was supported by a National Science Foundation Senior Postdoctoral Fellowship and a grant from the Science Research Council and enjoyed the hospitality of the University of Oxford and the Weizmann Institute of Science. He gratefully acknowledges the support of these institutions.

Tel-Aviv University

AND

UNIVERSITY OF CHICAGO 



\section{PACIFIC JOURNAL OF MATHEMATICS \\ EDITORS}

\author{
Richard ARENS (Managing Editor) \\ University of California \\ Los Angeles, California 90024
}

\author{
R. A. Beaumont \\ University of Washington \\ Seattle, Washington 98105
}

\section{J. DugundJI}

Department of Mathematics

University of Southern California

Los Angeles, California 90007

D. Gilbarg and J. Milgram

Stanford University

Stanford, California 94305

\section{ASSOCIATE EDITORS}
E. F. BECKENBACH
B. H. NeumanN
F. WOLF
K. YoshidA

\section{SUPPORTING INSTITUTIONS}

\author{
UNIVERSITY OF BRITISH COLUMBIA \\ CALIFORNIA INSTITUTE OF TECHNOLOGY \\ UNIVERSITY OF CALIFORNIA \\ MONTANA STATE UNIVERSITY \\ UNIVERSITY OF NEVADA \\ NEW MEXICO STATE UNIVERSITY \\ OREGON STATE UNIVERSITY \\ UNIVERSITY OF OREGON \\ OSAKA UNIVERSITY
}

\author{
UNIVERSITY OF SOUTHERN CALIFORNIA \\ STANFORD UNIVERSITY \\ UNIVERSITY OF TOKYO \\ UNIVERSITY OF UTAH \\ WASHINGTON STATE UNIVERSITY \\ UNIVERSITY OF WASHINGTON \\ AMERICAN MATHEMATICAL SOCIETY
}

The Supporting Institutions listed above contribute to the cost of publication of this Journal, but they are not owners or publishers and have no responsibility for its contents or policies.

Mathematical papers intended for publication in the Pacific Journal of Mathematics should be in typed form or offset-reproduced (not dittoed), double spaced with large margins. Underline Greek letters in red, German in green, and script in blue. The first paragraph or two must be capable of being used separately as a synopsis of the entire paper. Items of the bibliography should not be cited there unless absolutely necessary, in which case they must be identified by author and Journal, rather than by item number. Manuscripts, in duplicate, may be sent to any one of the four editors. Please classify according to the scheme of Math. Reviews, Index to Vol. 39. All other communications should be addressed to the managing editor, or Elaine Barth, University of California, Los Angeles, California, 90024.

100 reprints are provided free for each article, only if page charges have been substantially paid. Additional copies may be obtained at cost in multiples of 50 .

The Pacific Journal of Mathematics is issued monthly as of January 1966. Regular subscription rate: $\$ 72.00$ a year (6 Vols., 12 issues). Special rate: $\$ 36.00$ a year to individual members of supporting institutions.

Subscriptions, orders for back numbers, and changes of address should be sent to Pacific Journal of Mathematics, 103 Highland Boulevard, Berkeley, California, 94708.

PUBLISHED BY PACIFIC JOURNAL OF MATHEMATICS, A NON-PROFIT CORPORATION Printed at Jerusalem Academic Press, POB 2390, Jerusalem, Israel.

$$
\begin{gathered}
\text { Copyright (c) } 1975 \text { Pacific Journal of Mathematics } \\
\text { All Rights Reserved }
\end{gathered}
$$




\section{Pacific Journal of Mathematics}

Vol. 56, No. 2 December, 1975

Ralph Alexander, Generalized sums of distances .................. 297

Zvi Arad and George Isaac Glauberman, A characteristic subgroup of a group of odd order ............................... 305

B. Aupetit, Continuité du spectre dans les algèbres de Banach avec involution ........................................ 321

Roger W. Barnard and John Lawson Lewis, Coefficient bounds for some classes of starlike functions . ...........................

Roger W. Barnard and John Lawson Lewis, Subordination theorems for some classes of starlike fumctions . .................... 333

Ladislav Bican, Preradicals and injectivity .................. 367

James Donnell Buckholtz and Ken Shaw, Series expansions of analytic

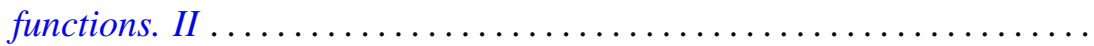

Richard D. Carmichael and E. O. Milton, Distributional boundary values in

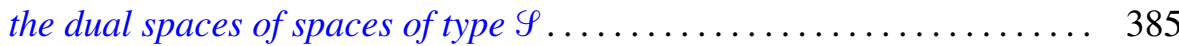

Edwin Duda, Weak-unicoherence ............................ 423

Albert Edrei, The Padé table of functions having a finite number of essential singularities ........................................

Joel N. Franklin and Solomon Wolf Golomb, A function-theoretic approach to the study of nonlinear recurring sequences ................ 455

George Isaac Glauberman, On Burnside's other $p^{a} q^{b}$ theorem 469

Arthur D. Grainger, Invariant subspaces of compact operators on topological vector spaces .........................

Jon Craig Helton, Mutual existence of sum and product integrals .

Franklin Takashi Iha, On boundary functionals and operators with finite-dimensional null spaces

Gerald J. Janusz, Generators for the Schur group of local and global number fields

A. Katsaras and Dar-Biau Liu, Integral representations of weakly compact operators.

W. J. Kim, On the first and the second conjugate points .

Charles Philip Lanski, Regularity and quotients in rings with involution ....

Ewing L. Lusk, An obstruction to extending isotopies of piecewise linear manifolds.

Saburou Saitoh, On some completenesses of the Bergman kernel and the Rudin kernel..

Stephen Jeffrey Willson, The converse to the Smith theorem for $Z_{p}$-homology spheres. 